\title{
Construction Engineering Safety Technology Based on X-ray Scanning BuXiangyi
}

Wuhan University of Technology, Wuhan 430070,China

\author{
Keywords: Construction engineering safety, Contraband, CF-1 type; Backscatter ;Construction \\ scanner security instrument
}

\begin{abstract}
X ray scanning technology has many unique advantages, which can fully meet the requirements of the security work of radiation safety. And it can effectively improve the efficiency of safety inspection to improve the level of prevention. From the back for fear of drug and construction safety inspection increasing emphasis point of view. This is a kind of market expectations are very optimistic about the technology, have a good prospect of industrialization.
\end{abstract}

\section{Introduction}

The current world and domestic back for fear of the situation is very grim, the terrorists have been the airport, rail transportation, large gatherings and other populated places as an important target of the attack, they use weapons to hijack a plane, and tried to use the sole, crotch bombs Dutch act explosive device to blow up the plane or innocent people. The terrorists have attacked many of terrorist attacks event. The assassination of heads of government human bomb used by terrorists, in India, Pakistan, Iraq and other countries, in the religious gatherings, large mart and shopping places to create a number of terrorist incidents, so that a large number of innocent people lost their lives. Christmas in 2009, the Nigeria youth Abdel put the PETN tied in the underpants trying to blow up a flight from Nigeria to Detroit flight America. The failed bombing attempt once again to bring attention to the airport security check, airports all over the world have strengthened security measures, based on traditional metal detection door, the installation of a new construction scanners ( $\mathrm{X}$ ray backscattering construction engineering safetyequipment), to detect whether passengers in the clothes inside harbor prohibited dangerous items.

\section{Human Safety Inspection Technology Development Present Situation}

The current practice in security, construction engineering safetyinspection equipment is mainly metal detection door and hand-held metal detector. The principle of the position limit, metal detection equipment can not accurately positioning tool metal of dangerous goods and details, more cannot detect contraband goods other than metal. The scope of the whole world to crack down on smuggling and aiming at an important place to carry out terrorist activities in full swing, strengthen the detection technology and equipment of contraband hiding on the high level of human needs of the people, therefore, the development of more convenient apparatus for human construction safety inspection technology effectively imminent. The following describes the metal detection, $\mathrm{THz}$ ray scanning detection, active and passive millimeter wave detection, $\mathrm{X}$ ray backscattering probe and $\mathrm{X}$ ray transmission line scanning technology is the main direction of research and current application of human security.

Metal DetectionTechnology. The security check door/handheld metal detectors by transmitting coil launch continuous wave or pulse wave, the establishment of an alternating magnetic field stability in the detection zone, when the metal into the detection zone, because the magnetic induction, the receiving coil receives the magnetic induction current, after amplification, adaptive circuit and the arithmetic logic circuit processing, to achieve the alarm. Its advantages are low cost, fast speed; the disadvantage is vulnerable to outside interference or strong metal electromagnetic field equipment, high false alarm rate; and detecting metal limited range, low detection sensitivity of nonferrous metals. 
This is an already popular using detection technology, the domestic related products include metal detection door, hand-held metal detectors, sole metal detector, widely used in airport, dock, station, large stadiums.

THz Detection Technology. THz rays generated at about $1000 \mathrm{GHz}$ frequency between microwave and infrared spectrum ranging from electromagnetic wave. Research shows that the $\mathrm{THz}$ electromagnetic radiation, a large penetration depth, no radiation damage to human tissue, high imaging resolution, image contrast and good uniformity. It can effectively identify of various conventional means cannot recognize the camouflage, stealth and deception measures in the optical spectrum. So far, several research units in North America and Europe, has developed the THz photoelectric imaging principle of various means of detection, and back in fear, counterfeit identification, postal system safety, chemical detection, airport security, biomedical optical imaging, metal and plastic landmine detection, aerospace vehicles with metal and composite material nondestructive flaw detection application level, showing a good potential application prospect.

Millimeter Wave Detection Technology. The millimeter wave frequency section in $30 \mathrm{GHz}$ to $300 \mathrm{GHz}$, the imaging of active and passive in two ways. The difference lies in the active mode needs a millimeter wave source, while passive way only need millimeter wave receiver. The main, passive millimeter wave can distance detection, real-time imaging, suspicious objects can detect hidden under the clothes; detection and visual effect, is suitable for fast imaging were detected, but the drawback is the specific species cannot distinguish suspicious things. As of early 2012, millimeter wave construction scanning imaging security inspection equipment has been in the United States and the European Union countries and an important airport facilities widely used, the installed capacity of the United States alone has nearly 600, the United States will be completed in 20141800 sets for deployment in the airport screeners.China has conducted a national major scientific instrument and equipment development of special "millimeter wave imaging detector development and industrialization demonstration" project to start.

$\mathrm{X}$ ray Detection Technology. X ray imaging detection technology is currently the security field, as well as one of the most commonly used means of checking contraband effective. $\mathrm{X}$ - ray security inspection equipment using the traditional transmission imaging technique, which can visually display the contents of the package, luggage, do not check box. In recent years, to adapt to changes in the international anti-terrorism situation, $\mathrm{X}$ - ray security inspection technologies in the field of the development of a new, $\mathrm{X}$ ray backscattering technique and transmission line scanning technology is one of the two most important study direction, caused a few domestic research units and equipment manufacturer's attention.

Backscattering technology is by issuing the $\mathrm{X}$ ray to the security object, and its acquisition from the scattered photon image to generate a backscattering detector. "Flying spot" scanning technology is the key to the implementation of the back scattering technique. "Flying spot scanning technology will $\mathrm{X}$ ray in instantaneous point form issued, shoot at the target, and the target happens Compton scattering. Back scattering technique can be effectively detected with low atomic number elements (such as carbon, hydrogen, oxygen, nitrogen, etc.) substances, such as explosives, plastics and other drugs. The back scattering detector to capture $\mathrm{X}$ ray photon generated image meets the security target scattering, show the image in the display of all backgrounds while can clear identification of suspicious items.

Backscattering technology applied to the human construction safety inspection equipment, can effectively and quickly detect hidden in the clothes of the construction under the all kinds of dangerous substances and contraband, its powerful function is a common metal detectors cannot, match again with imaging technology, security personnel can easily point to a specific position of dangerous substances or prohibited goods, thereby saving a large number of security of time, and eliminates the aggressive touch security or security of light.

Human construction $\mathrm{X}$ ray backscattering security equipment in foreign countries have a preliminary application. American AS\&E and Rapiscan two companies have been able to provide the X X-ray back scattering construction imaging security inspection equipment. 
From the foreign application reports of view, there are two main problems at present. The first is to check the image relates to personal privacy. On the other hand is the general public do not understand this technique, the existence of $\mathrm{X}$ ray fear. In fact, "flying spot scanning technology to make" amount of radiation of this equipment is very low, regardless of subject or security officers are absolutely safe, no harm to health will not.

Transmission line scanning technology. Transmission line scanning technique using a high X-ray high-voltage broad-spectrum $\mathrm{X}$ ray source of security objects emit $\mathrm{X}$ rays, and by line scanning the relative movement of the security object and the fan-shaped X-ray beam. Transmission line array detector, the use of security objects in different parts of the $\mathrm{X}$ - ray shows different attenuation coefficients of different gray, image generation.

The human construction $\mathrm{X}$ ray line scan security inspection technology, micro dose safety inspection into the field of human construction safety inspection, check the single dose of less than 5 uGy, less than the hospital chest X-ray doses of one percent. This equipment can clearly detect contraband in vivo to carry, can help the fight against terrorism and drug trafficking.

\section{CF-1 Type Back Scattering Construction Scanner Security Instrument}

Tianjin heavy party Technology Co. Ltd developed the CF-1 type back scattering construction scanner security instrument is a typical solution to $\mathrm{X}$ ray scanning technology based on construction engineering safetyscheme, it adopts the back scattering line scanning technology. As its main performance index,as Table 1.

Table 1 Main performance index of X optical back scattering detection equipment

\begin{tabular}{|l|l|l|}
\hline Index name & Test data & $\begin{array}{l}\text { Foreign data } \\
\text { (AS\&E) }\end{array}$ \\
\hline A single scan dose & $<0.1 \mathrm{uGy}$ & $0.1 \mathrm{uGy}$ \\
\hline $\begin{array}{l}\text { Equipment surface leakage } \\
\text { dose rate }\end{array}$ & $<2 \mathrm{uGy/h}$ & $2 \mathrm{uGy} / \mathrm{h}$ \\
\hline A single scan time & 8 seconds & 8 seconds \\
\hline Spatial resolution & $\begin{array}{l}0.8 \mathrm{~mm} \\
\text { copper wire }\end{array}$ & \begin{tabular}{l} 
wire \\
\hline
\end{tabular}
\end{tabular}

OutstandingCharacteristic. Doses of small. single scanning radiation dose of less than $0.1 \mathrm{uGy}$, is absolutely safe to human construction . Because the CF-1 security inspection equipment using flying spot scanning technology.The human construction only by the instantaneous dose of an $\mathrm{X}$ ray points, so a single detected radiation dose is very low, the comparative figures is simple, once security is nearly $1 / 1000$ of the radiation dose to a chest X-ray film,

Wide measuring range, high recognition rate. Not only can detect metal objects of high atomic number, more can detect non-metallic objects of low atomic number. Dangerous goods to people inside the clothes and construction surface throughout the concealed weapons, ammunition and drugs, ceramic knives, plastic bombs, gasoline, acetone etc. now the use of security equipment is difficult to perceive clearly detected.

CF-1 type back scattering construction scanner security caused by the flying spot scanning technology, $\mathrm{X}$ - ray equipment itself with very low leakage, equipment with remote control, for a long time, the security of operating personnel will not cause any harm.

For the image of private parts processing, will not lead to exposure of personal privacy concerns.

Practical Application of the Equipment for Example. CF-1 back scattering construction scanner security system is in terrorist attacks to the possible social each profession, the unit for the promotion, at present already in the detention center, rail transportation and other places has a very 
good application. Tianjin city Hongqiao district which is used for detecting the guards, specialized in and out of the prison inmates, police, visitors might be carrying drugs, suicide self destruct tools, dangerous goods, tobacco, communication tools and other prohibited items into the prison area, to provide a quick and efficient security means for prisoners prison, prison management, preventing terrorist attacks etc. CF-1 type back scattering construction scanner security instrument can completely replace the existing metal security door, hand held metal detector for human security, and can detect the metal detectors cannot detect contraband non-metallic dangerous goods, products, and its not detect the dead zone.

Faced to cope with the international and domestic anti-terrorism situation, effectively prevent and combat crime, security becomes more and more important, the governments investment in security equipment in rapid growth year after year. From 2010 to 2015 America security investment amount of more than \$39000000000, have installed CTX scanning device in $75 \%$ airports, and the implementation of security checks on passengers waiting bus system.

\section{Conclusion}

This paper provides an overview of the human construction as a carrier of terrorist activities, summarizes the status of the development of human safety inspection techniques. It Shows a heavy party technology by using of X-ray backscatter imaging scanning principle. By making micro-dose X-rays penetrate the construction 's clothing and spot hidden behind clothes fired objects, it has different backscatter intensity based on these different objects to accurately determine whether the prohibited items CF-1 type backscatter construction scanners security practical application of the detector device.

\section{References}

[1] Araujo, A.C., Viana, P.R.M., Peres, A.E.C.. Reagents in iron ores flotation [J]. Minerals Engineering, 2005,18 (2): 219-224.

[2] Yan, H.Z.,Gong, W.Q., Mei, G.J., et al. Evaluation of Biodegradability of Amine Collectors [J]. Advanced Materials Research, 2010, 113-116: 267-271.

[3] Ingvild, E.H., Odd, G.B., Karl, A.H.. Environmental impact of amines [J]. Energy Procedia, 2009 (1): 1297-1304.

[4] Araujo, D.M., Yoshida, M.I., Takahashi, J.A., et al. Biodegradation studies on fatty amines used for reverse flotation of iron ore [J]. International Biodeterioration and Biodegradation, 2010,64 (2): 151-155.

[5] Borls, N.A., Yolanda, M.C, Martin, A.. Effect of surfactants at low concentrations on the desorption and biodegradation of sorbed aromatic compounds in soil [J]. Environmental Science \& Technology. 1991, 25 (10): 1728-1731.

[6] International Standards Organization, ISO 7827-1984(E), Water Quality-Evaluation in an Aqueous Medium of the Ultimate Aerobic Biodegradability of Organic Compounds-Methods by Analysis of Dissolved Organic Carbon (DOC), International Standards Organization, 1984 Geneva.

[7] OECD, 301 B: CO2 evolution test. In: Guidelines for the testing of chemicals, Section 3 (Degradation and accumulation), (Ready biodegradability). Organisation for Economic Co-operation and Development, Paris,1993: 18-23.

[8] Van, G.C.G., Stroo, C.A., Kroon, A.G.M.. Biodegradability of ethoxylated fatty amines and amides and the non-toxicity of their biodegradation products [J]. Tenside Surfactants Detergents, 1993, 30 (3): 213-216. 